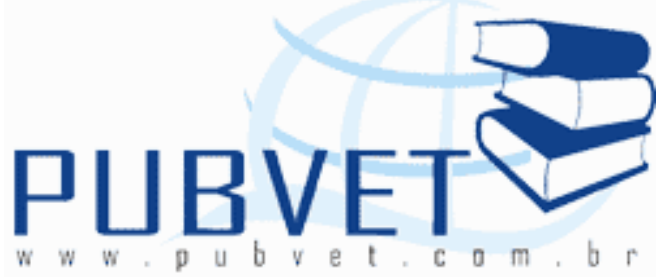

PUBVET, Publicações em Medicina Veterinária e Zootecnia.

\title{
Megaesôfago congênito em cão
}

Naira Moura Alves ${ }^{1}$, Tairon Pannunzio Dias e Silva ${ }^{2 *}$, Alécio Matos Pereira ${ }^{3}$, Juliana Rosito ${ }^{1}$, Mônica Mayrbaurl Borges ${ }^{4}$

${ }^{1}$ Médica Veterinária

${ }^{2}$ Médico Veterinário, Mestrando do Programa de Pós-Graduação em Zootecnia - UFPI/CPCE

${ }^{3}$ Médico Veterinário, Professor UFPI/CPCE, Doutorando do Programa de Pós Graduação em Ciência Animal

${ }^{4}$ Graduanda em Medicina Veterinária - UFPI/CPCE

*tairon.mvet@gmail.com

\section{Resumo}

Megaesôfago é o termo que se refere à dilatação e à hipomotilidade esofágica, resultante de distúrbio primário ou secundário. Os autores objetivaram relatar um caso de megaesôfago congênito em cão. Um cão macho, SRD, cinco meses de idade, foi encaminhado ao setor de Clínica Médica de Pequenos Animais do Hospital Veterinário da Universidade Federal do Piauí, apresentando fraqueza nos membros anteriores, regurgitações frequentes pelo nariz e boca logo após alimentação. Ao exame clínico o animal apresentava relutância em movimentar-se e corrimento nasal mucopurulento. Com base na anamnese, no exame físico e exame radiográfico foi confirmado um caso de megaesôfago congênito com acentuada dilatação esofágica a nível cervical e torácico com 
ALVES, N.M. et al. Megaesôfago congênito em cão. PUBVet, Londrina, V. 7, N. 23, Ed. 246, Art. 1627, Dezembro, 2013.

deslocamento ventral da traquéia e coração. Como terapia optou-se pela correção dietética com algumas adequações da postura do animal no momento e após a alimentação visando o trânsito normal do alimento e buscando eliminar a possibilidade de retenção alimentar e/ou regurgitações. A anamnese, os sinais clínicos aliados ao exame radiográfico confirmaram um caso de megaesôfago congênito em cão com melhora significativa da sintomatologia a partir da segunda semana do tratamento dietético conservador.

Palavras-chave: diagnóstico, fraqueza esofágica, regurgitação, tratamento.

\section{Congenital megaesophagus in dog}

\section{Abstract}

Megaesophagus is a term that refers to dilate the lower esophageal dysmotility and resulting disturbance primary or secondary. The authors aimed to report a case of congenital megaesophagus in a dog. A male dog, SRD, five months old, was referred to the sector of Internal Medicine Small Animal Veterinary Hospital of the Federal University of Piauí, with weakness in the limbs, frequent regurgitation through the nose and mouth after feeding. On clinical examination the dog was reluctant to move and purulent nasal discharge. Based on history, physical examination and radiographic examination confirmed a case of congenital megaesophagus with marked dilatation cervical and thoracic level with ventral displacement of the trachea and heart. As a therapy it was decided to fix with some dietary adjustments of posture of the animal at the time and after feeding, targeting the normal transit of food and trying to eliminate the possibility of withholding food and / or regurgitation. The history, clinical signs combined with the radiographic examination confirmed a case of congenital megaesophagus in dogs with symptoms improvement from the second week of dietary treatment conservative.

Keywords: diagnosis, weakness esophageal, regurgitation, treatment 
ALVES, N.M. et al. Megaesôfago congênito em cão. PUBVET, Londrina, V. 7, N. 23, Ed. 246, Art. 1627, Dezembro, 2013.

\section{INTRODUÇÃO}

O megaesôfago ou síndrome de megaesôfago denomina-se assim pela complexidade do seu quadro, com origens diversas, podendo ser definido como dilatação patológica do esôfago (TORRES, 1997). Uma destruição celular a níveis de $50 \%$ a $95 \%$ ocorre uma progressiva desorganização de toda a atividade motora e a dilatação do órgão (Celano et al., 2007).

O megaesôfago pode ser um distúrbio primário (congênito, idiopático ou adquirido) ou secundário a obstrução esofágica ou disfunção neuromuscular. 0 sinal clínico mais frequente é a regurgitação, acarretando em um quadro de perda de peso e caquexia. O tratamento até o presente momento, não é eficiente para a fraqueza esofágica, recorre-se a dieta pastosa para tentar evitar mais dilatação e pneumonia por aspiração (Nelson \& Couto, 2010).

A etiologia do megaesôfago congênito ainda é pouco conhecida, sugere-se que possa ser associado a uma falha sensorial ou uma lesão no centro de deglutição, mais especificamente na região medial da formação reticular lateral do tronco cerebral, com consequências no peristaltismo esofágico em animais jovens (Leib, 1983; Torres, 1997).

Esta patologia tem como predileção por cães das raças Fox Terriers, Schnauzers miniaturas, além de ser uma afecção hereditária também nas raças de Pastor Alemão, Newfoundland Dinamarquês Great Dane, Setter Irlandês, Shar Pei, Pug, Greyhound (Tilley \& Smith, 2008).

Os animais são geralmente trazidos ao veterinário por apresentarem regurgitação, associada ou não a perda de peso, tosse ou febre decorrente de pneumonia. Ocasionalmente, a tosse e outros sinais de traqueíte e/ou pneumonia por aspiração podem ser os únicos relatos do proprietário. No início da doença, a ocorrência de regurgitação de alimentos ocorre logo após sua ingestão, podendo ocorrer após minutos ou horas (Fossum et al., 1997).

A doença congênita, não adquirida, é suspeita quando a regurgitação e/ou a aspiração são observadas no animal ainda jovem. Se as características clínicas forem relativamente discretas ou intermitentes, o diagnóstico pode deixar de ser estabelecido até que o animal esteja mais velho, mas a análise 
ALVES, N.M. et al. Megaesôfago congênito em cão. PUBVET, Londrina, V. 7, N. 23, Ed. 246, Art. 1627, Dezembro, 2013.

do histórico deve sugerir que os sinais estavam presentes desde a infância. 0 diagnóstico é firmado através da análise dos sinais clínicos, por meio de anamnese, e como recurso adicional utiliza-se a esofagografia, onde se observa dilatação extensa do esôfago e deslocamento da traquéia e coração (Sturgess \& Dunn, 2001).

Atualmente, a fraqueza esofágica congênita não pode ser resolvida por meio do tratamento médico conservador, embora a administração de cisaprida $(0,25 \mathrm{mg} / \mathrm{kg})$ pareça, em alguns casos, melhorar os sinais. O manejo dietético conservador é usado na tentativa de prevenir maior dilatação e aspiração (Willard, 2006).

O prognóstico é dependente da causa e do tempo de ocorrência dos sintomas. Quanto mais cedo for identificada a patologia, maior a chance de sucesso no tratamento. Pode ser reservado, sendo que os pacientes com distúrbios adjacentes primários podem melhorar se tal distúrbio for tratado com êxito (Trindade, 2007). Ressalta-se o perigo causado pela pneumonia por aspiração e a importância da administração de alimentação especial (Tilley \& Smith, 2008). De posse dessas informações, os autores objetivaram relatar um caso de megaesôfago congênito em cão.

\section{CASO CLÍNICO}

Foi atendido um cão macho, SRD, cinco meses de idade, pesando $4,3 \mathrm{Kg}$, com o histórico de ter sido abandonado na porta da atual proprietária, registrado sob o RG: 1384, no setor de Clínica Médica de Pequenos Animais do Hospital Veterinário da Universidade Federal do Piauí. Segundo a proprietária, o animal apresentava fraqueza nos membros anteriores, vômitos frequentes pelo nariz e boca, a mesma relatou que isso acontecia sempre que o animal se alimentava ou realizava esforço.

Ao exame clínico, foi constatado emagrecimento, apatia, mucosas normocoradas, observou-se um estado nutricional de caquexia, todos os parâmetros fisiológicos dentro da normalidade, porém o animal apresentava relutância em movimentar-se e corrimento nasal mucopurulento (Figura 1A). 
ALVES, N.M. et al. Megaesôfago congênito em cão. PUBVet, Londrina, V. 7, N. 23, Ed. 246, Art. 1627, Dezembro, 2013.

Com base na anamnese e no exame físico foi levantada a suspeita de megaesôfago, com isso, foi solicitado exame radiográfico para avaliação cervical e torácica.

O exame radiográfico apresentou acentuada dilatação esofágica a nível torácico com deslocamento ventral da traquéia e coração (Figura 1B).

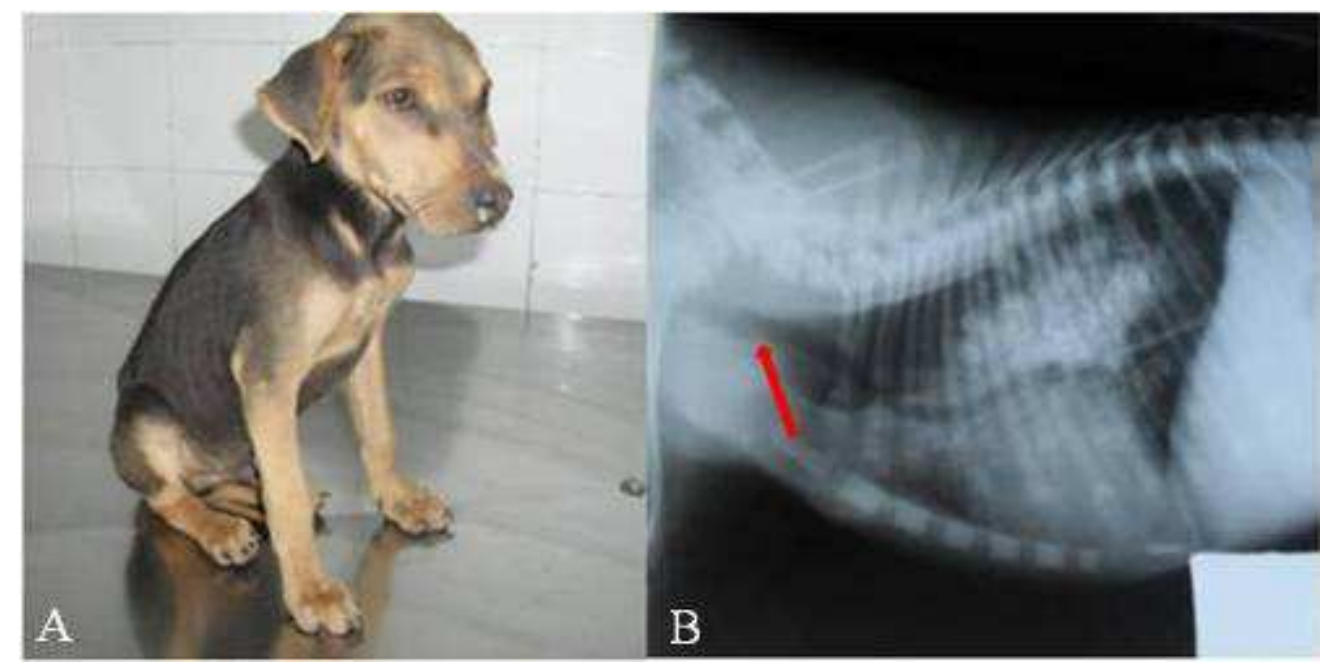

Figura 1: (A) Cão, SRD, cinco meses de idade, apresentando sinais clínicos característicos de megaesôfago. (B) Radiografia Simples: Presença de ar no lúmen esofágico torácico, deslocamento ventral da traquéia e do coração.

O diagnóstico definitivo foi obtido através de radiografia contrastada utilizando sulfato de bário como meio de contraste observando-se ampla dilatação esofágica a nível cervical e torácico com alterações de posicionamento traqueal e do coração (Figura 2). 


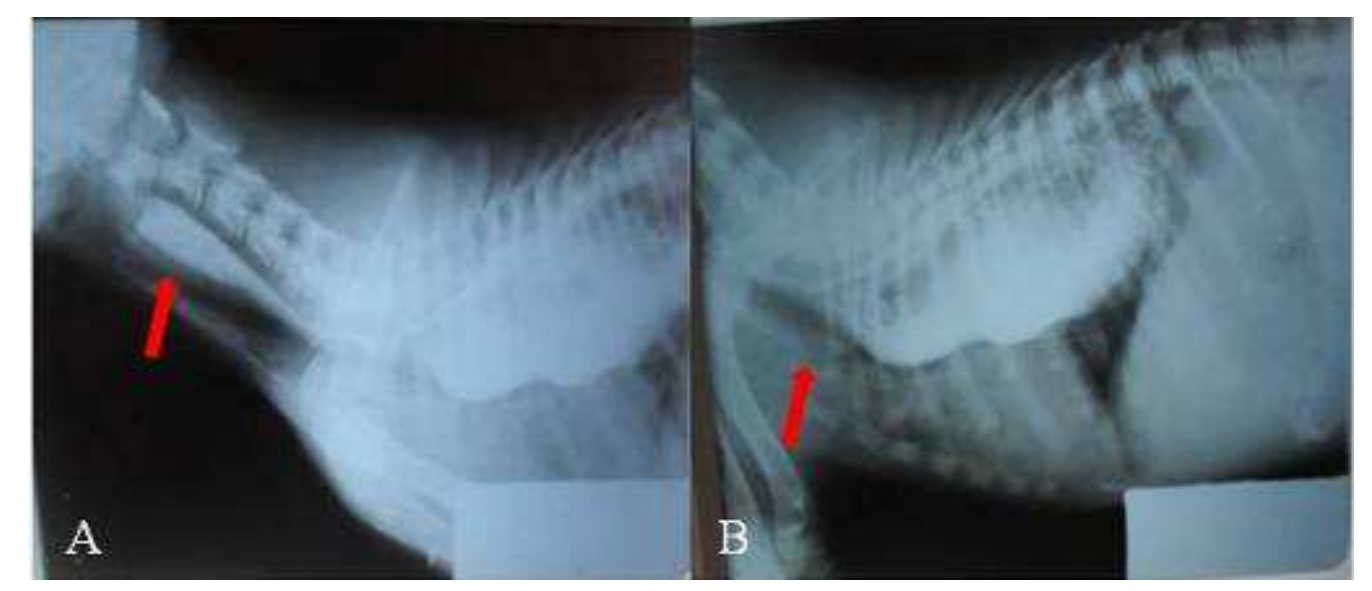

Figura 2. (A) Esofagografia cervical contrastada com sulfato de bário, dilatação esofágica. (B) Esofagografia torácica contrastada, mostrando alteração esofágica e suas decorrentes alterações.

Como terapia optou-se pela correção dietética com algumas adequações da postura do animal no momento e após a alimentação, visando o trânsito normal do alimento e buscando eliminar a possibilidade de retenção alimentar e/ou regurgitações. Foi prescrito o seguinte esquema dietético em quatro alimentações diárias:

$1^{\text {a }}$ semana: ração batida no liquidificador;

$2^{\text {a }}$ semana: arroz, verdura e carne, triturado no liquidificador;

$3^{a}$ semana: ração e patê, batido no liquidificador.

Uma hora antes da alimentação, administrar 0,3 mg/Kg Bromoprida e repetir $2 \mathrm{~h}$ depois. Durante a alimentação preconiza-se que o animal seja mantido em uma posição que favoreça a deglutição do alimento e após a alimentação ele seja mantido nessa posição por cinco a dez minutos.

\section{RESULTADOS E DISCUSSÃO}

De posse dos dados da anamnese, dos sinais clínicos e exames radiográficos, foi confirmado um caso de megaesôfago congênito e em compatibilidade com o que preconiza a literatura (Willard, 2006), foi prescrito o tratamento dietético conservador, obtendo a partir da segunda semana de tratamento resultados favoráveis ao paciente. 
ALVES, N.M. et al. Megaesôfago congênito em cão. PUBVET, Londrina, V. 7, N. 23, Ed. 246, Art. 1627, Dezembro, 2013.

É de extrema importância que esta alimentação assistida seja uma prática constante para animais com megaesôfago, uma vez que proporciona sua adaptabilidade às novas condições de alimentação oral para que o animal possa obter um escore corporal adequado, pois muitas complicações podem surgir em decorrência de um animal caquético e consequentemente imunossuprimido (German, 2005).

O caso relatado trata-se de megaesôfago de caráter congênito, visto que, o filhote apresentou os sinais clínicos sugestivos dessa patologia com apenas um mês de idade. A forma idiopática do megaesôfago é a causa mais comum, podendo ser congênita, sendo mais normalmente visto em filhotes, e a adquirida mais comum em animais idosos. A forma adquirida ocorre secundariamente a várias doenças sistêmicas como a miastenia grave (Andrande et al., 2007).

O megaesôfago congênito corresponde à hipomotilidade e à dilatação generalizada do esôfago, provocando regurgitação e subdesenvolvimento do filhote após o desmame. A patogenia da forma congênita ainda não está completamente esclarecida, embora estudos apontem para um defeito na inervação aferente vagal para o estômago (Washabau, 2004).

De acordo com German (2005) a principal manifestação clínica de megaesôfago é regurgitação. Mas essa regurgitação deve ser diferenciada de vômito, de náusea e de disfagia. A regurgitação caracteriza-se por uma eliminação retrógrada passiva do alimento não digerido a partir do esôfago. 0 vômito caracteriza-se pelas atividades coordenadas dos sistemas gastrintestinal, musculoesquelético e nervoso, culminando na eliminação ativa do alimento digerido ou parcialmente digerido pelo trato gastrintestinal.

De acordo com o relatado pela proprietária, foi possível afirmar que o animal supracitado apresentava regurgitação, pois o conteúdo apresentado caracteriza alimento não digerido e também pelo pequeno intervalo entre a alimentação e expulsão do conteúdo, considerando-se que o vômito sendo um conteúdo já digerido, requer um maior intervalo após a ingestão do alimento e ocorrência de tal evento (German, 2005). 
ALVES, N.M. et al. Megaesôfago congênito em cão. PUBVet, Londrina, V. 7, N. 23, Ed. 246, Art. 1627, Dezembro, 2013.

Na radiografia simples foi observada a dilatação esofágica, presença de ar no lúmen esofágico cervical e torácico, deslocamento ventral da traquéia e do coração (Sturgess \& Dunn, 2001) e pulmões sem nenhuma alteração morfológica aparente.

Embora a radiografia simples tenha mostrado bem evidente a dilatação esofágica, foi realizada a radiografia contrastada, utilizando sulfato de bário, comprovando então a existência do Megaesôfago. Segundo Spillmann (2007), a radiografia contrastada é indicada quando a radiografia simples não consegue definir o diagnóstico.

Até o momento, não há cura ou tratamento clínico que solucione a debilidade esofágica congênita. No caso em questão foi indicado um tratamento dietético conservador, a fim de evitar o agravamento da dilatação e possível aspiração de conteúdo (Tanaka et al. 2010).

Classicamente, o animal é submetido a uma alimentação pastosa, em uma plataforma elevada que requeira o animal em estação, com o apoio dos membros posteriores (Willard, 2006). Desta maneira, o esôfago cervical e torácico permanece em posição vertical quando o alimento é ingerido, o que permite que a gravidade auxilie a passagem do alimento através do esôfago para o estômago. Esta posição deve ser mantida por cinco a dez minutos após a alimentação. Oferecer várias refeições por dia em pequenas quantidades também evita a retenção de alimento no esôfago (Willard, 2006).

\section{CONCLUSÃO}

A anamnese, os sinais clínicos aliados ao exame radiográfico confirmaram um caso de megaesôfago congênito em cão com melhora significativa da sintomatologia a partir da segunda semana do tratamento dietético conservador. 


\section{REFERÊNCIAS BIBLIOGRÁFICAS}

ANDRADE, S. F; BARILLI; R. M. N; MELCHER; A; ET AL. Megaesôfago secundário à miastenia grave em uma cadela da raça Pastor Alemão. Semina: Ciências Agrárias, v. 28, n. 3, p. 477-482, 2007.

CELANO, R. M. G. et al. Avaliação nutricional pré-operatória dos pacientes com megaesôfago não-avançado. Revista do Colégio Brasileiro de Cirurgiões, v. 34, n. 3, p. 25-31, 2007.

FOSSUM, T.W. Cirurgia de pequenos animais. São Paulo: Roca, p. 286-87, 1997.

GERMAN, A. J. How treat megaesophagus. In: NORTH AMERICAN VETERINARY CONFERENCE, 19., 2005. Orlando. Proceedings... Orlando: North American Veterinary Conference, 2005.

LEIB, M. S. Megaesophagus in the dog, Part I, Anatomy, physiology and pathophysiology. Compendium of Continuous Education on Small Animal Practice, Auburn, v.5, n.10, p.825-833, 1983.

RICHARD W. NELSON; C.GUILLERMO COUTO. Medicina Interna de Pequenos Animais. 4 ed. Rio de Janeiro; p. 418 -419, 2010.

SPILLMANN, T. Esophageal diseases diagnostic and therapeutic approach. In: ANNUAL WSAVA CONGRESS, 32., 2007, Sydney. Proceedings... Sydney: Wsava Congress, 2007.

STURGESS, C.P.: DUNN, J.K. Tratado de Medicina de pequenos animais. São Paulo: Roca, p.385-87, 2001.

TANAKA, N.M.; HOOGEVONINK, N.; TUCHOLSKI, Â.P.; TRAPP[C], S.M.; FREHSE, M.S. Megaesôfago em cães. Rev. Acad., Ciênc. Agrár. Ambient., v.8, n.3, p.271-279, 2010.

TILLEY, L. P.; SMITH, F. W. K. Consulta veterinária em 5 minutos. 2. ed. Barueri: Manole, 2008.

TORRES, P. Megaesófago en el perro. Revisión bibliográfica y proposición de una nueva clasificación. Archivos de Medicina Veterinária, Valdivia, v.29, n.1, p.13- 23, 1997.

TRINDADE, R. L. Megaesôfago secundário a persistência de arco aórtico direito em um felino de 2 anos : Relato de caso: Universidade Castelo Branco Instituto Qualittas de Pósgraduação; 2007.

WASHABAU, R. J. Doenças do esôfago. In: ETTINGER, S. J.; FELDMAN, E. C. Tratado de medicina interna veterinária: doenças do cão e gato. 5. ed. Rio de Janeiro: Guanabara Koogan, 2004. p. 1205-1214.

WILLARD, M. D. Distúrbios da cavidade oral, faringe e esôfago. In: COUTO, C. G.; NELSON, R. W. Medicina interna de pequenos animais. 3. ed. Rio de Janeiro: Elsevier, 2006. p. 398399. 